Electroluminescence enhancement of SiGe/Si multiple quantum wells through nanowall structures

This article has been downloaded from IOPscience. Please scroll down to see the full text article. 2008 Nanotechnology 19365705

(http://iopscience.iop.org/0957-4484/19/36/365705)

The Table of Contents and more related content is available

Download details:

IP Address: 140.112.113.225

The article was downloaded on 04/03/2009 at 07:54

Please note that terms and conditions apply. 


\title{
Electroluminescence enhancement of SiGe/Si multiple quantum wells through nanowall structures
}

\author{
T T Chen ${ }^{1}$, Y P Hsieh ${ }^{1}$, C M Wei ${ }^{1}$, Y F Chen ${ }^{1}$, L-C Chen ${ }^{2}$, \\ K-H Chen ${ }^{2}$, Y H Peng ${ }^{3}$ and C H Kuan ${ }^{4}$ \\ ${ }^{1}$ Department of Physics, National Taiwan University, Taipei 106, Taiwan \\ ${ }^{2}$ Center for Condensed Matter Sciences, National Taiwan University, Taipei 106, Taiwan \\ ${ }^{3}$ Department of Electronic Engineering, Lan Yang Institute of Technology, I Lan 261, Taiwan \\ ${ }^{4}$ Department of Electrical Engineering and Graduate Institute of Electronics Engineering, \\ National Taiwan University, Taipei 106, Taiwan \\ E-mail: yfchen@phys.ntu.edu.tw
}

Received 18 May 2008, in final form 24 June 2008

Published 28 July 2008

Online at stacks.iop.org/Nano/19/365705

\begin{abstract}
The enhancement of light extraction from $\mathrm{Si}_{0.5} \mathrm{Ge}_{0.5} / \mathrm{Si}$ multiple quantum wells (MQWs) with nanowall structures fabricated by electron cyclotron resonance (ECR) plasma etching is presented. It is shown that the ECR plasma treatment does not damage the crystalline quality. At a driving current of $5.5 \times 10^{6} \mathrm{~A} \mathrm{~m}^{-2}$, the light output intensity of the MQWs with nanowall structures shows an enhancement of about $50 \%$ compared with that of the original MQWs. In addition to the enhanced light extraction, the improved optoelectronic properties are also attributed to the strain relaxation in nanowall structures.
\end{abstract}

\section{Introduction}

Silicon (Si)-based devices are highly attractive due to the well-known benefits of low cost, high reliability, and multifunctionality [1], which have made Si-based light-emitting diodes (LEDs) become one of the most important devices for optoelectronic integrated circuits. Notably, in recent years, a great deal of research on $\beta-\mathrm{FeSi}_{2}$ [2], erbium-doped $\mathrm{Si}$ [3], and SiGe alloys $[4,5]$ has been conducted due to their potential applications in fiber optical communication $(1.3-1.5 \mu \mathrm{m})$. Among these candidates, SiGe alloys have been investigated intensively. However, due to the nature of indirect band structure, SiGe-based devices are always considered as poor emitters. Hence, it is highly desirable to improve the efficiency of light-emission devices based on SiGe material.

In general, the light output of LEDs is a product of light internal efficiency and light extraction efficiency. According to a previous report, the light internal efficiency of SiGe epitaxial layers is only about $0.1 \%$ at $300 \mathrm{~K}$ [5]. Besides, the light extraction efficiency of Si-based LEDs is also low due to the large refractive index difference between $\mathrm{Si}$ (3.45) and air (1.0) [1], which results in total internal reflection inside the LEDs. According to Snell's law $\left[\theta_{\text {crit }}=\sin ^{-1}\left(n_{\text {air }} / n_{\mathrm{Si}}\right)\right]$, the critical angle is calculated to be approximately $17^{\circ}$. The light outside the critical angle is reflected from the interface, reabsorbed, and then confined internally. Assuming that light emitted from sidewalls and backside is neglected, the escape probability is about $2.2 \%$ from top surface of the LEDs.

To improve the light extraction efficiency, several approaches have been implemented for GaAs and III-nitridebased LEDs, such as surface roughening of top surface of capping layer or indium-tin-oxide contact by etching processes [6-8], photonic crystal structures [9], polymer film imprinting [10], and micro-lenses [11]. However, for Sibased optical devices, there are only a few studies denoted to the improvement of the light extraction efficiency, such as the rugged $\mathrm{SiN}_{x}$ pattern formed on the top of Si-based LEDs [12]. In this paper, we demonstrate that the nanowall structures fabricated by electron cyclotron resonance (ECR) plasma etching on the top surface of $\mathrm{Si}_{0.5} \mathrm{Ge}_{0.5} / \mathrm{Si}$ multiple quantum wells (MQWs) can be used to greatly enhance the light output intensity compared with that of MQWs without ECR plasma treatment [13]. In addition, the underlying mechanism has been illustrated, which may serve as the foundation for the application of the result of our approach to many other materials. 


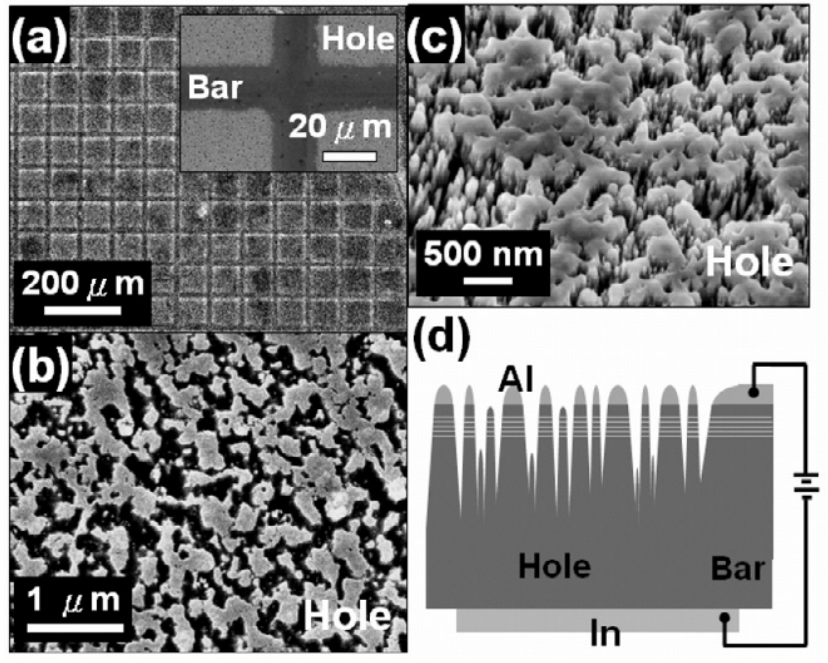

Figure 1. Scanning electron microscopy images of (a), (b) top view, and (c) tilt angle of $45^{\circ}$. (d) Schematic of the MQWs with nanowall structures.

\section{Experiment}

The $\mathrm{Si} / \mathrm{Si}_{0.5} \mathrm{Ge}_{0.5}$ MQWs studied were grown on $n^{+}(100)$ $\mathrm{Si}(0.0018 \Omega)$ substrate by a commercial ultrahigh-vacuum chemical vapor deposition system. The growth temperature was maintained at $600^{\circ} \mathrm{C}$ by a vacuum annealing furnace. The sample consists of a $100 \mathrm{~nm} \mathrm{Si} \mathrm{buffer} \mathrm{layer,} \mathrm{a} \mathrm{series} \mathrm{of} \mathrm{five}$ periods of $4 \mathrm{~nm}$ thick $\mathrm{Si}_{0.5} \mathrm{Ge}_{0.5}$ wells and $16 \mathrm{~nm}$ thick $\mathrm{Si}$ barriers, and a $100 \mathrm{~nm} \mathrm{Si} \mathrm{capping} \mathrm{layer.} \mathrm{All} \mathrm{of} \mathrm{the} \mathrm{MQWs}$ in this study were cut from the same base wafer.

To create nanowall structures on the MQWs, an aluminum (Al) contact layer of $50 \mathrm{~nm}$ was first deposited on the top surface of the MQWs by a vacuum evaporator system (ULVAC). Then, a gold made square-mesh grid (bought from Electron Microscopy Sciences Company) was placed on the Al-coated MQWs. An ECR plasma reactor was used to fabricate the nanowall structures. During the etching process, a gas mixture consisting of argon ( $\mathrm{Ar}$ ) and hydrogen $\left(\mathrm{H}_{2}\right)$ was included in the ECR plasma. The detailed etching process can be found in our previous report [13]. The thin Al coating layer will partly be melted and reassembled, which then forms a random masked pattern; after etching, the nanowall structures were thus obtained. After the etching process and removing the square-mesh grid, it was found that the area of MQWs covered by the gold bars was almost unetched, as shown in figure 1(a). For the area of holes, there were nanowall structures and the $\mathrm{Al}$ contact still remained on the top of nanowall structures, as shown in figures 1 (b) and (c). The nanowall structures are about $200 \mathrm{~nm}$ in width and $500 \mathrm{~nm}$ in depth. The depth of nanowall structures was measured by atomic force microscopy (AFM) measurement (not show here). Figure 1(d) shows a diagram of the MQWs with nanowall structures.

In order to reduce the number of surface defects created during the formation of nanowall structures, the samples were treated by thermal annealing at different temperatures. For the best thermal annealing conditions, the sample was placed in a quartz furnace and immediately heated to $450^{\circ} \mathrm{C}$ with

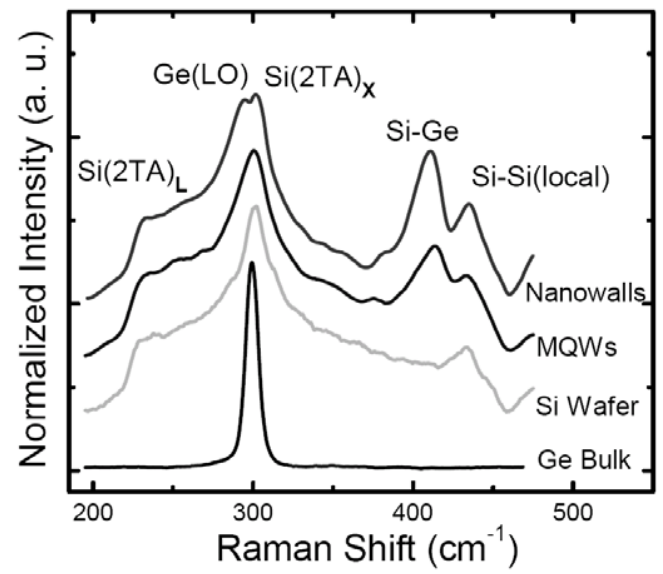

Figure 2. Raman scattering spectra of Si wafer, Ge bulk, and MQWs with and without nanowall structures.

a ramping rate of about $30^{\circ} \mathrm{C} \mathrm{min}^{-1}$ from room temperature at first, and then heated to $550^{\circ} \mathrm{C}$ with a ramping rate of $5^{\circ} \mathrm{C} \mathrm{min}{ }^{-1}$. After being maintained at $550^{\circ} \mathrm{C}$ for $30 \mathrm{~min}$, the sample was allowed to cool naturally to room temperature. The sample in the furnace was under an Ar environment with a pressure of 40 pounds per square inch and a flow rate of $300 \mathrm{sccm}$.

For comparison, the same square-mesh grid mask was used to form the mesh pattern of the aluminum contact on another identical MQW without ECR plasma treatment. Finally, both samples were annealed at $650^{\circ} \mathrm{C}$ for $1 \mathrm{~min}$ to form the ohmic contacts, and indium (In) was used as the contact layer on the back surface.

Scanning electron microscopy (SEM) images were recorded with a JEOL-JSM 6500 system. Micro-Raman scattering measurement was performed at room temperature in a backscattering geometry with a Jobin-Yvon T64000 system working in the triple-subtractive mode. An argonion laser working at $514.5 \mathrm{~nm}$ was used as the excitation source. Current-voltage $(I-V)$ characteristics were measured using a Keithley 236 source measurement unit. For the electroluminescence (EL) measurement, the sample was placed inside a closed-cycle helium (He) cryostat, and a Keithley 236 unit was used as the power supply. The EL signal was recorded with a Spectra Pro 300i monochromator, and detected by an InGaAs detector with an appropriate filter.

\section{Results and discussion}

In order to analyze the crystal quality of the sample after ECR plasma treatment, we performed micro-Raman measurements, as shown in figure 2. For the Si wafer, the frequencies at $231 \mathrm{~cm}^{-1}, 302 \mathrm{~cm}^{-1}$, and $434 \mathrm{~cm}^{-1}$ are respectively assigned to $\mathrm{Si}(2 \mathrm{TA})_{\mathrm{L}}, \mathrm{Si}(2 \mathrm{TA})_{\mathrm{X}}$, and $\mathrm{Si}-\mathrm{Si}($ local $)$ phonon modes. For the Ge bulk, the peak at $300 \mathrm{~cm}^{-1}$ is assigned to the $\mathrm{Ge}(\mathrm{LO})$ phonon mode. For the MQWs with and without nanowall structures, the $\mathrm{Si}(2 \mathrm{TA})_{\mathrm{X}}$ and $\mathrm{Ge}(\mathrm{LO})$ phonon modes cannot be distinguished, and the feature at $413 \mathrm{~cm}^{-1}$ is assigned to the $\mathrm{Si}-\mathrm{Ge}$ phonon mode [14]. It is found that the $\mathrm{Si}(2 \mathrm{TA})_{\mathrm{L}}$ 


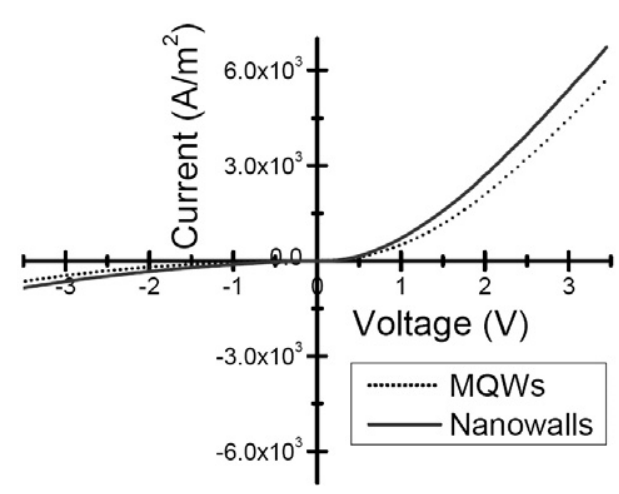

Figure 3. $I-V$ characteristics of MQWs with and without nanowall structures.

and $\mathrm{Si}-\mathrm{Si}$ (local) phonon modes are almost unchanged, which implies that these phonon modes arise from the Si substrate. For the MQWs with nanowall structures, both the Si-Ge and $\mathrm{Ge}(\mathrm{LO})$ phonon modes show a red-shift with respect to that of the original MQWs. This may arise from the strain relaxation in nanowall structures. When the SiGe layer is embedded in the MQWs, it experiences a compressive strain and the phonon modes are blue-shifted. After the formation of nanowall structures by the ECR plasma treatment, the compressive strain is relaxed, and the phonon modes will be red-shifted toward the original values [14]. The strength of the strain can be estimated by the following expression: $\omega_{\mathrm{Si}-\mathrm{Ge}}=400.5+14.2 x-575 \varepsilon$, where $\omega_{\mathrm{Si}-\mathrm{Ge}}$ is the frequency of the $\mathrm{Si}-\mathrm{Ge}$ phonon mode, $x$ the Ge content, and $\varepsilon$ the strength of biaxial strain [14]. Because both samples have the same Ge content of 0.5 , the estimated $\varepsilon$ in the MQWs with and without the nanowall structures is respectively about $-0.59 \%$ and $-0.94 \%$. It is stressed here that both samples reveal a compressive biaxial strain in the SiGe layers, and the strength of the strain has been reduced by about $0.35 \%$ in the MQWs with nanowall structures. Besides, the linewidth of the Si-Ge phonon mode in the MQWs with nanowall structures $\left(\sim 27.5 \mathrm{~cm}^{-1}\right)$ is narrower than that of the original MQWs $\left(\sim 38.5 \mathrm{~cm}^{-1}\right)$, which implies that the crystalline quality of the sample with nanowall structures was not damaged by the etching process. The narrower linewidth after the ECR plasma treatment may be attributed to the reduction of internal strain which improves the uniformity of the MQWs. The intensity of the $\mathrm{Si}-\mathrm{Ge}(\mathrm{Ge}(\mathrm{LO}))$ phonon mode is enhanced in the MQWs with nanowall structures as compared with that in the original MQWs, which may arise from the fact that some of the SiGe material is now directly exposed to the incident light.

Figure 3 compares the $I-V$ characteristics of the MQWs with and without nanowall structures. We can see that the turnon threshold voltage of the MQWs with nanowall structures is slightly lower than that of the original MQWs. This result may be interpreted in terms of the geometric effect, in which the nanowall structures have a sharp shape on the top, as shown in figure 1(d), while the original MQW has a flat surface. The sharp structure will have a stronger electric field on the top of the nanowall structures at the same forward bias, which then causes a lower threshold voltage. Besides, the reverse leakage

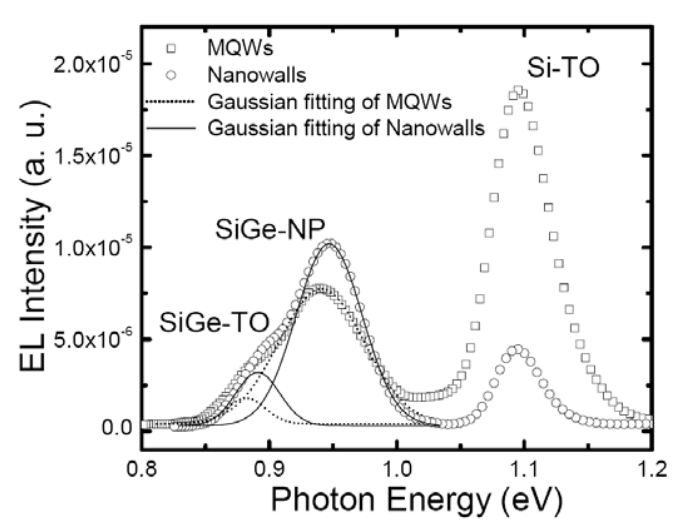

Figure 4. Electroluminescence spectra of the MQWs with and without nanowall structures at the same injection current of $4.6 \times 10^{6} \mathrm{~A} \mathrm{~m}^{-2}$ at $15 \mathrm{~K}$

currents of these two samples are almost identical, which again suggests that the etching process does not significantly degrade the electrical properties of the MQWs with nanowall structures.

Figure 4 shows the EL spectra for the MQWs with and without nanowall structures taken at the same injection current density of $4.6 \times 10^{6} \mathrm{~A} \mathrm{~m}^{-2}$ at $15 \mathrm{~K}$. The high and low peak features can be respectively attributed to the TO-phonon replica of $\mathrm{Si}(\mathrm{Si}-\mathrm{TO})$ and the type-II transitions of $\mathrm{Si}_{0.5} \mathrm{Ge}_{0.5} / \mathrm{Si}$ MQWs. The type-II transitions can be well fitted via two Gaussian curves, which contain a no-phonon transition (SiGeNP) and a TO-phonon replica of the no-phonon transition (SiGe-TO) $[4,5]$. It is found that the EL intensity of the type-II transitions is stronger than that of the Si-TO for the MQWs with nanowall structures. However, the MQW without ECR plasma treatment exhibits a reverse behavior. This may be due to the fact that the sidewall area of SiGe layers for light extraction has been greatly enhanced in the MQWs with nanowall structures, as shown in figure $1(\mathrm{~d})$. Besides, for the MQWs with nanowall structures, the obviously reduced EL intensity of the Si-TO may be attributed to the reabsorption of $\mathrm{Si}-\mathrm{TO}$ by $\mathrm{Si}_{0.5} \mathrm{Ge}_{0.5}$ layers due to the light trapping of nanowall structures. Additionally, the greater etching volume of the $\mathrm{Si}$ layers than that of the $\mathrm{Si}_{0.5} \mathrm{Ge}_{0.5}$ layers as shown in figure 1(d) may also contributed to the reduction of the Si-TO emission.

In contrast to the unchanged EL peak position of Si-TO, the peak position of the type-II transitions shows a clear blueshift. This can also be well interpreted in terms of the strain relaxation in the MQWs with nanowall structures similar to the red-shift of the phonon modes in the Raman spectra as shown in figure 2. The relaxation of the biaxial strain will increase both of the band gap energy of $\mathrm{Si}$ and $\mathrm{Si}_{0.5} \mathrm{Ge}_{0.5}$ layers [15], which is responsible for the observed blue-shift of the EL peak position. To have a more quantitative analysis, let us adopt the calculated result in previous report [15]. The strain relaxation induced band gap energy shifts of $\mathrm{Si}$ and $\mathrm{Si}_{0.5} \mathrm{Ge}_{0.5}$ layers are respectively calculated to be 182 and $113 \mathrm{meV} / \%$ strain. The band gap energy of the type-II transition can be calculated through the following equation: $E_{\text {type-II }}=E_{\mathrm{SiGe}}-\Delta E_{\mathrm{c}}=E_{\mathrm{SiGe}}-\left(E_{\mathrm{Si}}-E_{\mathrm{SiGe}}\right) \times Q_{\mathrm{c}}$, 


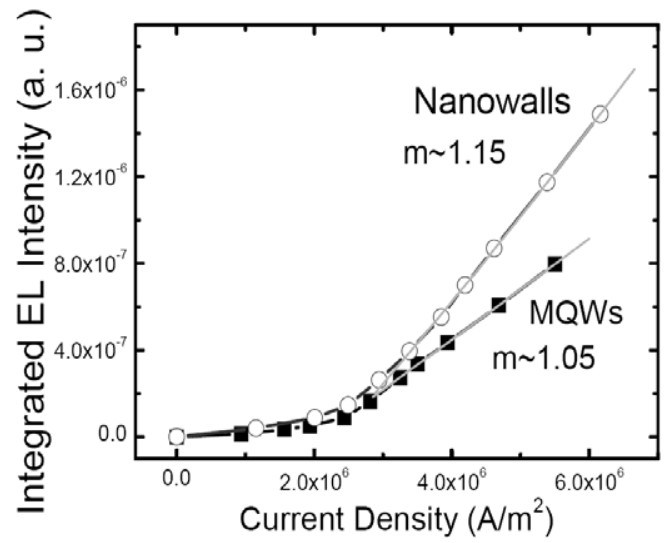

Figure 5. Integrated electroluminescence intensity of the type-II transitions as a function of forward current at $15 \mathrm{~K}$.

where $\Delta E_{\mathrm{c}}$ is the conduction band offset, $E_{\mathrm{Si}}\left(E_{\mathrm{SiGe}}\right)$ the band gap energy of $\mathrm{Si}(\mathrm{SiGe})$ layers, and $Q_{\mathrm{c}}$ the type-II conduction band offset ratio of 0.3 [16]. Through the calculation, the strain relaxation induced band gap energy shift of the typeII transitions is about $30 \mathrm{meV}$ when the strain is reduced from $0.94 \%$ to $0.59 \%$. This value is on the same order of the magnitude with the blue-shift of the EL peak position as shown in figure 4. Additionally, the narrower linewidth of the type-II transitions of the MQWs with nanowall structures again provides an additional piece of evidence supporting our interpretation that the change in the EL spectra is due to the strain relaxation after ECR plasma treatment. Because of the strain relaxation, the energy variation due to strain is greatly reduced.

The current dependent integrated EL intensities of the type-II transitions of these samples measured at $15 \mathrm{~K}$ were obtained via the Gaussian fitting curves, as shown in figure 5. We can see that the integrated EL intensity increases with increasing injection current. It is found that both curves show a linear dependence for the forward current exceeding $3 \times 10^{6} \mathrm{~A} \mathrm{~m}^{-2}$. The dependence can be characterized by $L \sim$ $I^{m}$, where $L$ is the integrated EL intensity, $I$ is the injection current, and the exponent $m$ can be used to characterize the emission mechanism. As shown in figure 5, the exponent $m$ for the MQWs with and without nanowall structures is respectively about 1.15 and 1.05, which indicates that most of the injected carriers recombine radiatively $[4,5]$. The steeper rise of the $L-$ $I$ relationship indicates that the quantum efficiency of the EL process in the MQWs with nanowall structures is higher than that of the MQWs without ECR plasma treatment.

\section{Conclusion}

In conclusion, we have demonstrated that ECR plasma treatment provides an alternative method to enhance the light extraction from $\mathrm{Si}_{0.5} \mathrm{Ge}_{0.5} / \mathrm{Si}$ MQWs. Our results indicate that the crystalline quality of the MQWs was not damaged through the etching process. It is found that after ECR plasma treatment, the optoelectronic properties can be improved substantially due to strain relaxation in addition to the enhanced light extraction. Notably, the measured light output intensity of the MQWs with nanowall structures shows an increase up to about 1.5 times compared with that of the MQWs without ECR plasma treatment.

\section{Acknowledgments}

This work was supported by the Ministry of Education and National Science Council of the Republic of China.

\section{References}

[1] Levinshtein M E, Rumyantsev S L and Shur M S 2001 Properties of Advanced Semiconductor Materials (UK: Wiley) p 173

[2] Leong D, Harry M, Reeson K J and Homewood K P 1997 Nature 387686

[3] Ishiyama T, Yoneyama S, Yamashita Y, Kamiura Y, Date T, Hasegawa T, Inoue K and Okuno K 2006 Physica B 376/377 122

[4] Apetz R, Vescan L, Hartmann A, Dieker C and Lüth H 1995 Appl. Phys. Lett. 66445

[5] Stoica T and Vescan L 2003 Semicond. Sci. Technol. 18409

[6] Horng R H, Yang C C, Wu J Y, Huang S H, Lee C E and Wuu D S 2005 Appl. Phys. Lett. 86221101

[7] Huang H-W, Chu J T, Kao C C, Hseuh T H, Lu T C, Kuo H C, Wang S C and Yu C C 2005 Nanotechnology 161844

[8] Cao M, Lao Y, Wu H, Liu C, Xie Z, Cao C and Wu H 2008 J. Vac. Sci. Technol. A 26219

[9] Kim T, Danner A J and Choquette K D 2005 Electron. Lett. 411138

[10] Wang Z M et al 2007 Semicond. Sci. Technol. 22279

[11] Lee M K, Ho C L and Fan C H 2008 Appl. Phys. Lett. 92061103

[12] Kim K-H, Shin J-H, Park N-M, Huh C, Kim T-Y, Cho K-S, Hong J C and Sung G Y 2006 Appl. Phys. Lett. 89191120

[13] Hsu C-H, Lo H-C, Chen C-F, Wu C T, Hwang J-S, Das D, Tsai J, Chen L-C and Chen K-H 2004 Nano Lett. 4471

[14] Baranov A V, Fedorov A V, Perova T S, Moore R A, Yam V, Bouchier D, Thanh V Le and Berwick K 2006 Phys. Rev. B 73075322

[15] Brunner K 2002 Rep. Prog. Phys. 6527

[16] Cheng H H, Yen S T and Nicholas R J 2000 Phys. Rev. B 624638 